SECTION 21. Pedagogy. Psychology. Innovations in the field of education.

Yunona Rinatovna Krakhmaleva candidate of technical Sciences, associate Professor of Mathematics,

Taraz state University named after M.Kh. Dulati, Kazakhstan

yuna_kr@mail.ru

Gulzhan Kadyrkhanovna Dzhanabayeva master 2 year degree in Mathematics

Taraz state University named after M.Kh. Dulati, Kazakhstan

\title{
METHODOLOGICAL BASIS FOR SOLVING SYSTEMS OF DIFFERENTIAL EQUATIONS IN MAPLE
}

\begin{abstract}
For solving systems of differential equations with fixed factor with the application of computer algebra system Maple is given. Method of the matrix exponential for homogeneous systems is shown.

Key words: The system of differential equations, the method of the matrix exponential, matrix, eigenvalues, eigenvectors. Attached vectors.
\end{abstract}

\section{УДК 372.851.2}

\section{МЕТОДИЧЕСКИЕ ОСНОВЫ РЕШЕНИЯ СИСТЕМ ЛИНЕЙНЫХ ДИФФЕРЕНЦИАЛЬНЫХ УРАВНЕНИЙ В МАРLЕ}

Аннотация: Рассматривается применение системы компьютерной алгебры Maple для решения систем дифференциальных уравнений с постоянными коэффициентами. Показывается метод матричной экспоненты для однородных систем.

Ключевые слова: Система дифференциальных уравнений, метод матричной экспоненты, матрица, собственные значения, собственные векторы. присоединенные векторы.

Методы решения линейных уравнений и систем совершенствовались на протяжении всего 19 века, и как известно решающее влияние оказали успехи алгебры. Выяснение аналогий между линейными алгебраическими и линейными дифференциальными уравнениями явилось исходным пунктом символических методов, начиная с Бриссона и Коши. Создание Вейрштрассом теории элементарных делителей позволило вместе с Жорданом построить общую теорию линейных систем уравнений с постоянными коэффициентами.

На современном этапе важным для приложений является экономные методы нахождение аналитического решения дифференциальных уравнений. В связи с этим, с каждым днем возрастает роль быстро развивающейся символьной или компьютерной математики, в спектр задач математических пакетов которой, входит проведение математических исследований, требующих вычислений и аналитических выкладок.

Представим методику решения системы линейной дифференциальной однородной системы с постоянными коэффициентами

используя метод матричной экспоненты .

$$
\dot{x}=P x,
$$

При решении дифференциальных систем необходимы знания линейной алгебры для того, чтобы выполнить матрично-векторные выкладки, что занимает достаточно много времени. В связи с этим, представляемая методика решения дифференциальных систем 
подразумевает интенсивное использование средств линейной алгебры. Сеанс работы с MAPLE начинается с введением команды restart:

$>$ restart; with (linalg):

Рассмотрим методику решения линейной однородной дифференциальной системы с постоянными коэффициентами на примере, где матрица коэффициентов имеет различные действительные собственные значения $\lambda$ :

$$
\left\{\begin{array}{l}
\frac{d x}{d t}=3 x-y+z, \\
\frac{d y}{d t}=-x+5 y-z, \\
\frac{d z}{d t}=x-y+3 z
\end{array}\right.
$$

Решаемая система имеет вид

$$
\dot{X}=A \cdot X(t),
$$

где

$$
A=\left(\begin{array}{ccc}
3 & -1 & 1 \\
-1 & 5 & -1 \\
1 & -1 & 3
\end{array}\right), X(t)=\left(\begin{array}{l}
x(t) \\
y(t) \\
z(t)
\end{array}\right), \dot{X}=\left(\begin{array}{l}
\frac{d x}{d t} \\
\frac{d y}{d t} \\
\frac{d z}{d t}
\end{array}\right) .
$$

Вводится матрица $A$ и определяется единичная матрица размерности $3 \times 3$, которая нужна для вычисления собственных значений матрицы $A$ :

$>A:=\operatorname{matrix}([[3,-1,1],[-1,5,-1],[1,-1,3]]) ; I 3:=\operatorname{diag}(1,1,1)$;

$$
\begin{aligned}
& A:=\left[\begin{array}{rrr}
3 & -1 & 1 \\
-1 & 5 & -1 \\
1 & -1 & 3
\end{array}\right] \\
& I 3:=\left[\begin{array}{lll}
1 & 0 & 0 \\
0 & 1 & 0 \\
0 & 0 & 1
\end{array}\right]
\end{aligned}
$$

Для вычисления собственных значений и собственных векторов матрицы коэффициентов нужно вычислить определитель $\operatorname{det}(A-\lambda I)$ :

$>Q 0:=\operatorname{charpoly}(\mathrm{A}$, lambda) ;

$$
Q 0:=\lambda^{3}-11 \lambda^{2}+36 \lambda-36
$$

$>$ solve $(Q 0=0)$;

$$
6,2,3
$$

Находим собственные векторы матрицы $A$ :

$>$ eigenvectors (A);

$$
[3,1,\{[1,1,1]\}],[2,1,\{[-1,0,1]\}],[6,1,\{[1,-2,1]\}]
$$

Каждый из этих списков соответствует одному собственному значению. Сначала указывается само собственное значение, затем его кратность, затем в фигурных скобках перечисляются собственные векторы. Запомним эти векторы:

$>e 1:=\operatorname{vector}([1,1,1]) ; e 2:=\operatorname{vector}([-1,0,1]) ; e 3:=\operatorname{vector}([1,-2,1])$;

$$
e l:=[1,1,1]
$$




\section{Impact Factor $($ ISI $)=0.307$ based on International Citation Report (ICR)}

$$
\begin{aligned}
& e 2:=[-1,0,1] \\
& e 3:=[1,-2,1]
\end{aligned}
$$

B MAPLE существуют специальные команды для сборки матриц по столбцам, которые мы используем:

$>\mathrm{P}:=$ augment $(\mathrm{e} 1, \mathrm{e} 2, \mathrm{e} 3) ; \mathrm{J}:=\operatorname{diag}(3,2,6)$;

$$
\begin{gathered}
P:=\left[\begin{array}{rrr}
1 & -1 & 1 \\
1 & 0 & -2 \\
1 & 1 & 1
\end{array}\right] \\
J:=\left[\begin{array}{llr}
3 & 0 & 0 \\
0 & 2 & 0 \\
0 & 0 & 6
\end{array}\right]
\end{gathered}
$$

Фундаментальная матрица решений однородной системы находится по формуле $H(t)=P \cdot e^{J t}:$

$>\mathrm{H}:=\operatorname{evalm}(\mathrm{P \&}$ exponential $(\mathrm{J}, \mathrm{t}))$;

$$
H:=\left[\begin{array}{ccc}
\mathbf{e}^{(3 t)} & -\mathbf{e}^{(2 t)} & \mathbf{e}^{(6 t)} \\
\mathbf{e}^{(3 t)} & 0 & -2 \mathbf{e}^{(6 t)} \\
\mathbf{e}^{(3 t)} & \mathbf{e}^{(2 t)} & \mathbf{e}^{(6 t)}
\end{array}\right]
$$

Транспонируем матрицу $H(t)$ :

$>\mathrm{F}:=$ transpose $(\mathrm{H})$;

$$
F:=\left[\begin{array}{ccc}
\mathbf{e}^{(3 t)} & \mathbf{e}^{(3 t)} & \mathbf{e}^{(3 t)} \\
-\mathbf{e}^{(2 t)} & 0 & \mathbf{e}^{(2 t)} \\
\mathbf{e}^{(6 t)} & -2 \mathbf{e}^{(6 t)} & \mathbf{e}^{(6 t)}
\end{array}\right]
$$

И теперь, зная фундаментальную матрицу решений записываем решения дифференциальной системы:

$>\mathbf{F F}:=\operatorname{Matrix}($ row $(F, 1))$;

$$
F F:=\left[\begin{array}{lll}
\mathbf{e}^{(3 t)} & \mathbf{e}^{(3 t)} & \mathbf{e}^{(3 t)}
\end{array}\right]
$$

$>C 1:=\operatorname{Matrix}(3,1,[C[1], C[2], C[3]])$;

$$
C 1:=\left[\begin{array}{l}
C_{1} \\
C_{2} \\
C_{3}
\end{array}\right]
$$

$>\mathrm{X}[\mathrm{t}]:=\mathrm{FF} . \mathrm{C} 1$

$$
X_{t}:=\left[\mathbf{e}^{(3 t)} C_{1}+\mathbf{e}^{(3 t)} C_{2}+\mathbf{e}^{(3 t)} C_{3}\right]
$$

$>$ FD: =Matrix $(\operatorname{row}(F, 2))$;

$$
F D:=\left[-\mathbf{e}^{(2 t)} \quad 0 \quad \mathbf{e}^{(2 t)}\right]
$$

$>C 2:=\operatorname{Matrix}(3,1,[C[1], C[2], C[3]])$;

$$
C 2:=\left[\begin{array}{l}
C_{1} \\
C_{2} \\
C_{3}
\end{array}\right]
$$

$>\mathrm{Y}[\mathrm{t}]:=\mathrm{FD} . \mathrm{C} 2$;

$$
Y_{t}:=\left[-\mathbf{e}^{(2 t)} C_{1}+\mathbf{e}^{(2 t)} C_{3}\right]
$$

$>$ FG:=Matrix $(\operatorname{row}(F, 3))$; 


$$
F G:=\left[\mathbf{e}^{(6 t)}-2 \mathbf{e}^{(6 t)} \mathbf{e}^{(6 t)}\right]
$$

$>C 3:=\operatorname{Matrix}(3,1,[C[1], C[2], C[3]])$;

$$
C 3:=\left[\begin{array}{l}
C_{1} \\
C_{2} \\
C_{3}
\end{array}\right]
$$

$>\mathrm{Y}[\mathrm{t}]:=\mathrm{FG} . \mathrm{C} 3$

$$
Y_{t}:=\left[\mathbf{e}^{(6 t)} C_{1}-2 \mathbf{e}^{(6 t)} C_{2}+\mathbf{e}^{(6 t)} C_{3}\right]
$$

Методика решения линейной однородной дифференциальной системы с постоянными коэффициентами, где матрица коэффициентов имеет кратные действительные собственные значения $\lambda$ рассмотрим на примере такой системы:

$$
\left\{\begin{array}{l}
\dot{x}=2 x+y+z \\
\dot{y}=-2 x-z \\
\dot{z}=2 x+y+2 z
\end{array}\right.
$$

Решаемая система имеет вид

$$
\dot{X}=A \cdot X(t),
$$

где

$$
A=\left(\begin{array}{ccc}
2 & 1 & 1 \\
-2 & 0 & -1 \\
2 & 1 & 2
\end{array}\right), X(t)=\left(\begin{array}{l}
x(t) \\
y(t) \\
z(t)
\end{array}\right), \dot{X}=\left(\begin{array}{l}
\frac{d x}{d t} \\
\frac{d y}{d t} \\
\frac{d z}{d t}
\end{array}\right) .
$$

Вводим идентичные операции для рассматриваемой системы, описанные в методике решения линейной однородной дифференциальной системы с постоянными коэффициентами , где матрица коэффициентов имеет различные действительные собственные значения $\lambda$, вплоть до нахождения собственных значений :

$>$ eigenvectors (A);

$$
[2,1,\{[1,-2,2]\}],[1,2,\{[0,-1,1]\}]
$$

В данном случае система MAPLE сообщает, что собственное значение $\lambda=2$ имеет кратность $m=1$ и собственный вектор $(1,-2,2)^{E}$, а собственное значение $\lambda=1$ имеет кратность $m=2(1,-2,1)^{E}$ и только один собственный вектор $(0,-1,1)^{E}$. Запоминаем эти вектора:

$$
\begin{gathered}
>\text { e1:=vector }([0,-1,1]) ; e 2:=\operatorname{vector}([1,-2,2]) ; \\
e 1:=[0,-1,1] \\
e 2:=[1,-2,2]
\end{gathered}
$$

Собственный вектор $e_{1}$, соответствующий собственному значению $\lambda=1$ должен иметь один присоединенный вектор $p_{1}$, удовлетворяющий уравнению $\operatorname{det}(A-\lambda I) \cdot p_{1}=e_{1}$. Находим присоединенный вектор $p_{1}$ :

$>A A:=e v a l m(A-I 3) ; Q:=$ linsolve $(A A, e 1)$; 


$$
\begin{gathered}
A A:=\left[\begin{array}{rrr}
1 & 1 & 1 \\
-2 & -1 & -1 \\
2 & 1 & 1
\end{array}\right] \\
Q:=\left[1,-1-t_{1}, t_{1}\right]
\end{gathered}
$$

Компьютерная система выдает ответ с параметр _ $t_{1}$, который вправе выбирать по своему усмотрению. Положим $t_{1}=1$ :

$>Q:=[1,-1-t[1],-t[1]]$;

$\operatorname{subs}(t t[1] \overline{=1}, Q)$;

$$
\begin{aligned}
& Q:=\left[1,-1-t_{1},-t_{1}\right] \\
& \quad[1,-2,1]
\end{aligned}
$$

И запомним получившийся присоединенный вектор:

$>$ p1:=vector $([1,-2,1])$;

$$
p 1:=[1,-2,1]
$$

Теперь составляем согласно теории дифференциальных систем составляем жорданову форму, которая будет иметь две клетки:

$$
\begin{gathered}
A=P \cdot J \cdot P^{-1}, \\
P=\left[e_{1}, \mathrm{p}_{1}, \mathrm{e}_{2}\right], \\
J=\left[\begin{array}{cc}
J_{2}(1) & 0 \\
0 & J_{1}(2)
\end{array}\right] .
\end{gathered}
$$

Вводим специальные команды для формирования жордановых клеток и уже известную команду сборки матриц по столбцам:

$>\mathrm{P}:=\operatorname{augment}(\mathrm{e} 1, \mathrm{p} 1, \mathrm{e} 2) ; \mathrm{J}:=\mathrm{diag}(\operatorname{JordanBlock}(1,2), \operatorname{JordanBlock}(2,1))$;

$$
\begin{gathered}
P:=\left[\begin{array}{rrr}
0 & 1 & 1 \\
-1 & -2 & -2 \\
1 & 1 & 2
\end{array}\right] \\
J:=\left[\begin{array}{lll}
1 & 1 & 0 \\
0 & 1 & 0 \\
0 & 0 & 2
\end{array}\right]
\end{gathered}
$$

Проверим правильность приведения матрицы к жордановой форме:

$>\operatorname{evalm}\left(P \& \star J \& * P^{\wedge}(-1)\right)$;

$$
\left[\begin{array}{rrr}
2 & 1 & 1 \\
-2 & 0 & -1 \\
2 & 1 & 2
\end{array}\right]
$$

Результат, как видно, совпадает с матрицей коэффициентов $A$. Зная жорданову форму, нетрудно по известной формуле записать матричную экспоненту $e^{J t}$, для этого используем специальную функцию:

> eJt:=exponential $(J, t)$;

$$
e J t:=\left[\begin{array}{ccc}
\mathbf{e}^{t} & t \mathbf{e}^{t} & 0 \\
0 & \mathbf{e}^{t} & 0 \\
0 & 0 & \mathbf{e}^{(2 t)}
\end{array}\right]
$$

И теперь уже записывается фундаментальная матрица решений однородной системы $H(t)=P \cdot e^{J t}:$

$>\mathrm{H}:=\operatorname{evalm}(\mathrm{P} \& * \mathrm{eJt})$; 


$$
H:=\left[\begin{array}{ccc}
0 & \mathbf{e}^{t} & \mathbf{e}^{(2 t)} \\
-\mathbf{e}^{t} & -t \mathbf{e}^{t}-2 \mathbf{e}^{t} & -2 \mathbf{e}^{(2 t)} \\
\mathbf{e}^{t} & t \mathbf{e}^{t}+\mathbf{e}^{t} & 2 \mathbf{e}^{(2 t)}
\end{array}\right]
$$

Транспонирую матрицу $H$ получаем фундаментальную матрицу решений: $>$ F : =transpose $(\mathrm{H})$;

$$
F:=\left[\begin{array}{ccc}
0 & -\mathbf{e}^{t} & \mathbf{e}^{t} \\
\mathbf{e}^{t} & -t \mathbf{e}^{t}-2 \mathbf{e}^{t} & t \mathbf{e}^{t}+\mathbf{e}^{t} \\
\mathbf{e}^{(2 t)} & -2 \mathbf{e}^{(2 t)} & 2 \mathbf{e}^{(2 t)}
\end{array}\right] .
$$

Последний шаг - записывается решения исходной дифференциальной системы.

Методика решения линейной однородной дифференциальной системы с постоянными коэффициентами, где матрица коэффициентов имеет комплексные собственные значения $\lambda$ не отличается от методики решения линейной однородной дифференциальной системы с постоянными коэффициентами , в которой матрица коэффициентов имеет различные действительные собственные значения $\lambda$.Отличие возникает при формировании фундаментальной матрицы решений, которая в рассматриваемом случае является комплексной. Для того, чтобы результат был вещественным, используют свойство фундаментальной матрицы: любой столбец фундаментальной матрицы является частным решением. Тогда, любая комбинация любых ее столбцов также будет являться решением, которую можно заменить одним из участвующих в комбинировании столбцов. Имея в виду, что действительную и мнимую части комплексного числа можно получить комбинированием его самого и сопряженного к нему применяют эти известные факты для столбцов фундаментальной матрицы. Заменяя столбцы комплексной фундаментальной матрицей вновь полученными векторами, получают полностью вещественную фундаментальную матрицу решений.

Данная методика позволяет находить аналитические решения при любом числе входящих уравнений (и неизвестных функций ) в систему дифференциальных уравнений , что является ее достоинством, но при этом следует учесть, что приходиться вводить операции для упрощения рациональных и иррациональных выражений. Примером этому служит нахождение решения для следующей системы:

$$
\left\{\begin{array}{l}
\dot{x}=2 x-y+z+2 w \\
\dot{y}=x+2 y-z+w \\
\dot{z}=x-y+2 z+w \\
\dot{w}=x+z+w .
\end{array},\right.
$$

у которой собственные значения (кроме действительных, иррациональные числа) и соответствующие собственные векторы матрицы коэффициентов :

$>$ eigenvectors (P) ;

$$
\begin{aligned}
& {[2+\sqrt{2}, 1,\{[\sqrt{2}, 1,1,1]\}],[2-\sqrt{2}, 1,\{[-\sqrt{2}, 1,1,1]\}],[0,1,\{[-1,0,0,1]\}],} \\
& {\left[3,1,\left\{\left[\frac{5}{3}, 2,1, \frac{4}{3}\right]\right\}\right]}
\end{aligned}
$$

Фундаментальная матрица имеет вид: 


$F:=\left[\begin{array}{ccccc}-\frac{2 \mathbf{e}^{((2+\sqrt{2}) t)}}{(-2+\sqrt{2})(1+\sqrt{2})(2+\sqrt{2})(\sqrt{2}-1)} & \frac{\sqrt{2} \mathbf{e}^{(-(-2+\sqrt{2}) t)}}{(-2+\sqrt{2})(1+\sqrt{2})} & -1 & \frac{5}{3} \mathbf{e}^{(3 t)} \\ -\frac{2 \mathbf{e}^{((2+\sqrt{2}) t)}}{(-2+\sqrt{2})(1+\sqrt{2})(2+\sqrt{2})(\sqrt{2}-1)} & -\frac{\sqrt{2} \mathbf{e}^{(-(-2+\sqrt{2}) t)}}{(-2+\sqrt{2})(1+\sqrt{2})} & 0 & 2 \mathbf{e}^{(3 t)} \\ -\frac{2 \mathbf{e}^{((2+\sqrt{2}) t)}}{(-2+\sqrt{2})(1+\sqrt{2})(2+\sqrt{2})(\sqrt{2}-1)} & -\frac{\sqrt{2} \mathbf{e}^{(-(-2+\sqrt{2}) t)}}{(-2+\sqrt{2})(1+\sqrt{2})} & 0 & \mathbf{e}^{(3 t)} \\ -\frac{2 \mathbf{e}^{((2+\sqrt{2}) t)}}{(-2+\sqrt{2})(1+\sqrt{2})(2+\sqrt{2})(\sqrt{2}-1)} & -\frac{\sqrt{2} \mathbf{e}^{(-(-2+\sqrt{2}) t)}}{(-2+\sqrt{2})(1+\sqrt{2})} & 1 & \frac{4}{3} \mathbf{e}^{(3 t)}\end{array}\right]$

После введения преобразований фундаментальная матрица имеет более упрощенный вид, нежели раннее:

$f:=\operatorname{matrix}(4,4,[\operatorname{simplify}(F[1,1]), \operatorname{simplify}(F[1,2]), F[1,3], F[1,4]$, si mplify $(F[2,1])$, simplify $(F[2,2]), F[2,3], F[2,4], \operatorname{simplify}(F[3,1])$, si mplify $(F[3,2]), F[3,3], F[3,4], \operatorname{simplify}(F[4,1]), \operatorname{simplify}(F[4,2]), F[$ $4,3], \mathrm{F}[4,4]])$;

$$
f:=\left[\begin{array}{cccc}
\mathbf{e}^{((2+\sqrt{2}) t)} & \frac{\sqrt{2} \mathbf{e}^{(-(-2+\sqrt{2}) t)}}{(-2+\sqrt{2})(1+\sqrt{2})} & -1 & \frac{5}{3} \mathbf{e}^{(3 t)} \\
\mathbf{e}^{((2+\sqrt{2}) t)} & -\frac{\sqrt{2} \mathbf{e}^{(-(-2+\sqrt{2}) t)}}{(-2+\sqrt{2})(1+\sqrt{2})} & 0 & 2 \mathbf{e}^{(3 t)} \\
\mathbf{e}^{((2+\sqrt{2}) t)} & -\frac{\sqrt{2} \mathbf{e}^{(-(-2+\sqrt{2}) t)}}{(-2+\sqrt{2})(1+\sqrt{2})} & 0 & \mathbf{e}^{(3 t)} \\
\mathbf{e}^{((2+\sqrt{2}) t)} & -\frac{\sqrt{2} \mathbf{e}^{(-(-2+\sqrt{2}) t)}}{(-2+\sqrt{2})(1+\sqrt{2})} & 1 & \frac{4}{3} \mathbf{e}^{(3 t)}
\end{array}\right]
$$

\section{References:}

1. Бибиков Ю.Н. Курс обыкновенных дифференциальных уравнений,Москва, Высшая школа, 1991.

2. Эльсгольц Л.Э. Дифференциальные уравнения и вариационное исчисление.,М.,2000.

3. Федорюк М.В. Обыкновенные дифференциальные уравнения., М., 1980.

4. Карташев Э.А., Рождественский Б.Л. Обыкновенные дифференциальные уравнения и основы вариационные исчисления., М.,1976

5. Алексеев Е. Решение задач вычислительной математики в пакетах Mathcad 12, MATLAB 7, Maple 9, 2006 г.

6. Савченко С Е., Кузьмичева Т.Г., Методы решения задач в Maple, М., Наука, Физматлит, 2005

7. Баландин М.Ю.,Системы дифференциальных уравнений с постоянными с постоянными коэффициентами, Новосибирск: НГТУ, 2004

8. Е. Алексеев «Решение задач вычислительной математики в пакетах Mathcad 12, MATLAB 7, Maple 9», 2006 г. 